\title{
Abordagem fisioterapêutica nas disfunções sexuais femininas: uma revisão
}

\section{integrativa}

\author{
Physiotherapeutic approach in female sexual dysfunctions: an integrative review \\ Enfoque fisioterapéutico en las disfunciones sexuales femeninas: una revisión integrativa
}

Recebido: 29/10/2021 | Revisado: 01/11/2021 | Aceito: 05/11/2021 | Publicado: 07/11/2021

\author{
Sílvia Letícia Costa Prates \\ ORCID: https://orcid.org/0000-0003-0324-3019 \\ Centro Educacional da Amazonia, Brasil \\ E-mail: leticiacostaprates@gmail.com \\ Celinara Queiroz da Silva \\ ORCID: https://orcid.org/0000-0003-1771-0597 \\ Centro Educacional da Amazonia, Brasil \\ E-mail: celinara.portel6@gmail.com \\ Wanny Turriel do Nascimento \\ ORCID: https://orcid.org/0000-0002-8912-6966 \\ Centro Educacional da Amazonia, Brasil \\ E-mail: wturriel2019@gmail.com \\ Eliane Ferreira Marinho \\ ORCID: https://orcid.org/0000-0002-3323-6293 \\ Universidade Estadual do Pará, Brasil \\ E-mail: elianefmarinho@yahoo.com.br
}

\begin{abstract}
Resumo
Objetivo: Abordar as principais disfunções sexuais femininas, e as condutas fisioterapêuticas, enfatizando a importância para melhor qualidade de vida das mulheres tratadas. Metodologia: Trata-se de uma revisão integrativa, realizada em seis fases do processo de produção, através de artigos extraídos de periódicos, disponíveis nas bases de dados eletrônicas, como google acadêmico, scielo, Lilacs, Bireme (Biblioteca Virtual em Saúde-BVS). Seguindo os critérios de inclusão e exclusão, utilizou-se instrumento previamente elaborado, para se assegurar que os dados relevantes fossem extraídos, servindo como registro, para posterior análise dos dados. Resultados: Foram encontrados 31 trabalhos publicados, sendo selecionados 9, por apresentar maior aproximação com os objetivos propostos no estudo. Revelaram que as disfunções sexuais femininas estão presentes em grande parte da população feminina. Dentre as queixas mais comuns estão os distúrbios da excitação, do desejo hipoativo, anorgasmia, dispareunia, vaginismo, vulvodínia e dor pélvica crônica. Foram relacionadas as principais intervenções fisioterapêuticas, como a cinesioterapia, trabalho manual, biofeedback e eletroestimulação. O tratamento proporciona melhora da saúde sexual, maior autoconsciência, autoconfiança, melhora da imagem corporal e diminuição da ansiedade. Considerações Finais: O tratamento de disfunções sexuais muitas vezes é negligenciado, pela falta de conhecimento ou excesso de pudor. Mas, apesar da relutância ou preconceito, há um avanço significativo na discussão acerca do tema. A fisioterapia torna-se cada vez mais uma alternativa na hora de tratar desordens de origem uroginecológicas, sendo de extrema relevância para a melhor qualidade de vida das mulheres, incluindo a sexual.
\end{abstract}

Palavras-chave: Disfunção sexual feminina; Condutas fisioterapêuticas; Sexualidade feminina.

\begin{abstract}
Objective: Address the main female sexual dysfunctions, and physical therapy behavior, emphasizing the importance for a better quality of life for treated women. Methodology: This is an integrative review, carried out in six phases of the production process, through articles extracted from journals, available in electronic databases, such as academic google, scielo, Lilacs, Bireme (Virtual Health Library-VHL). Following the inclusion and exclusion criteria, a previously elaborated instrument was used to ensure that the relevant data were extracted, serving as a record for further data analysis. Results: Thirty-one published works were found, and 9 were selected as they were closer to the objectives proposed in the study. They revealed that female sexual dysfunctions are present in a large part of the female population. Among the most common complaints are disorders of arousal, hypoactive desire, anorgasmia, dyspareunia, vaginismus, vulvodynia, and chronic pelvic pain. The main physical therapy interventions were listed, such as kinesiotherapy, manual work, biofeedback, and electrical stimulation. The treatment provides improved sexual health, greater selfawareness, self-confidence, improved body image and reduced anxiety. Final Considerations: The treatment of sexual dysfunctions is often neglected, due to lack of knowledge or excessive modesty. But despite the reluctance or prejudice, there is a significant advance in the discussion on the subject. Physical therapy is increasingly becoming an alternative
\end{abstract}


when it comes to treating urogynecological disorders, being extremely relevant for a better quality of life for women, including sexual ones.

Keywords: Female sexual dysfunction; Physiotherapeutic conducts; Female sexuality.

\section{Resumen}

Objetivo: Abordar las principales disfunciones sexuales femeninas y conductas fisioterapéuticas, enfatizando la importancia de una mejor calidad de vida para las mujeres tratadas. Metodología: Se trata de una revisión integradora, realizada en seis fases del proceso de producción, a través de artículos extraídos de revistas, disponibles en bases de datos electrónicas, como google académico, scielo, Lilacs, Bireme (Biblioteca Virtual en Salud-BVS). Siguiendo los criterios de inclusión y exclusión, se utilizó un instrumento elaborado previamente para asegurar que se extrajeron los datos relevantes, sirviendo como registro para un posterior análisis de los datos. Resultados: Se encontraron 31 trabajos publicados y se seleccionaron 9 por estar más cerca de los objetivos propuestos en el estudio. Revelaron que las disfunciones sexuales femeninas están presentes en gran parte de la población femenina. Entre las quejas más comunes se encuentran los trastornos de la excitación, el deseo hipoactivo, la anorgasmia, la dispareunia, el vaginismo, la vulvodinia y el dolor pélvico crónico. Se enumeraron las principales intervenciones de fisioterapia, como kinesioterapia, trabajo manual, biorretroalimentación y estimulación eléctrica. El tratamiento proporciona una mejor salud sexual, mayor autoconciencia, autoconfianza, mejor imagen corporal y reducción de la ansiedad. Consideraciones finales: El tratamiento de las disfunciones sexuales a menudo se descuida por falta de conocimiento o modestia excesiva. Pero, a pesar de las reticencias o los prejuicios, hay un avance significativo en la discusión sobre el tema. La fisioterapia se está convirtiendo cada vez más en una alternativa a la hora de tratar los trastornos uroginecológicos, siendo de extrema relevancia para una mejor calidad de vida de las mujeres, incluidas las sexuales.

Palabras clave: Disfunción sexual femenina; Conductas fisioterapéuticas; Sexualidad femenina.

\section{Introdução}

A disfunção sexual feminina (DSF) compreende por toda a circunstância onde a mulher não consegue finalizar uma relação sexual ou em que esta seja insatisfatória para si e/ou para o seu companheiro por sentir desconforto ou dor na hora do ato (Ribeiro et al., 2013).

Por razões culturais o sexo até há algum tempo era visto somente como algo ligado a reprodução, o prazer era reprimido, por ser considerado pecaminoso ou moralmente condenável. Hoje, o sexo é parte do cotidiano das pessoas não estando limitado à concepção, já que o prazer humano independe da reprodução (Costa, 2011).

A sexualidade deve ser vivida de forma igualitária pelo homem e pela mulher, e o desfrutar de uma vida sexual boa e saudável vai propiciar felicidade e bem-estar (Milanese et al., 2020).

O funcionamento sexual é influenciado por quatro determinantes: o contexto biológico, o psicológico, o social (incluindo cultura) e as interações entre eles, por isso é crescente o reconhecimento do papel desempenhado pelo contexto social, especialmente a possibilidade de um parceiro sexual e a qualidade do relacionamento com ele (Fleury, 2015).

A falta de conhecimento e desinformação sobre a fisiologia da resposta sexual, medicamentos, condições uroginecológicas patológicas, problemas de ordem pessoal e, conflitos conjugais podem desencadear sérios problemas emocionais nas mulheres e consequentemente resultar em alguma disfunção sexual (Antonioli \& R Simões, 2010).

As disfunções sexuais afetam a qualidade de vida de diversas mulheres ocasionando constrangimento, incômodo, dores, mal-estar e uma vida sexual infeliz. Dentre algumas das principais disfunções sexuais estão:

\section{Vaginismo}

O vaginismo é uma patologia caracterizada pela contração involuntária da musculatura pélvica ocasionando dor vaginal e desconforto durante a penetração seja por ato sexual, toque, procedimentos ginecológicos, ou qualquer tipo de penetração. Sua procedência é dada por infecções frequentes, traumas sexuais, fatores psicológicos, evolução de dispareunia e por irritações sobre a vulva e a vagina. Sua análise ainda é complexa por falta de um conhecimento profissional suficiente e por ser de difícil abordagem, pois seus sintomas provocam grande incomodo e receio. (Moreira, 2013) 


\section{Dispareunia}

A dispareunia é o termo que caracteriza dor na localidade da vagina ligada ao ato da penetração. Pode estar relacionada ao vaginismo, dependendo da sua causa. Sua diferença para o vaginismo é que ocorre em grande parte por fatores hormonais e psicológicos. Costuma acontecer de maneira frequente e recorrente, gerando diversos problemas no dia a dia e na vida sexual da mulher (Soares, 2013)

\section{Anorgasmia}

É uma das disfunções sexuais mais recorrentes no sexo feminino, se trata da dificuldade ou incapacidade de ter orgasmo mesmo quando há interesse e disposição para o ato sexual. Pode ocorrer de forma primaria quando a mulher nunca teve nenhuma experiencia orgásmica ou de forma secundaria quando a mulher já passou pela experiência orgásmica, porém, passou a ter dificuldade ou incapacidade. Na forma primaria decorre normalmente por fatores psicológicos, cultura, costumes religiosos, escassez sobre educação social entre outros. Na forma secundaria decorre geralmente por um trauma físico ou psíquico. (Dias, 2015)

\section{Distúrbio da excitação}

O Distúrbio da excitação é quando há uma insuficiência frequente para sustentar e manter a excitação sexual, trazendo desconforto e constrangimento na mulher. Está ocasionada a uma ausência psicológica de excitação ou por escassez de lubrificação genital. (De Castro, 2020)

\section{Vulvodínia}

É uma dor crônica específica associada à hipersensibilidade no local da vulva, que pode ser provocada pelo contato ou espontaneamente onde a paciente tem como sintomas ardor, queimação, irritação ou prurido. A dor pode estar presente constantemente, ocorrendo com um leve desconforto ou dor intensa que irá limitar a mulher a sentar e se deitar. (Latorre et al., 2015).

\section{Dor pélvica crônica (DPC)}

É caracterizada como dor intensa na região inferior do abdômen ou região pélvica por um período de pelo menos 6 meses, podendo estar relacionada ou não com o período menstrual, ou relação sexual (Felipe, 2020)

\section{Transtorno de desejo sexual hipoativo (TDSH)}

Trata se de um distúrbio psicológico ou por uso de substâncias que provocam falta de desejo sexual, dificultando ou impossibilitando o ato, pode estar presente no início da vida sexual, após um período vivenciado ou de forma mista onde estão presentes tanto fatores psicológicos quanto ao uso de substâncias. (De Castro, 2020).

É importante ressaltar que a incidência das disfunções é difícil de ser determinada visto que muitas mulheres não procuram atendimento. Em contrapartida, as que se mobilizam e vão a uma consulta, têm encontrado poucas opções de tratamento, não incentivando as que não buscam (Antonioli \& R Simões, 2010).

Segundo Souza, (2020) os principais objetivos da fisioterapia nesta área são: a sensibilização e propriocepção dos músculos, a conscientização da contração e relaxamento muscular, normalizar o tônus muscular, aumentar a elasticidade na abertura vaginal, dessensibilizar áreas dolorosas, e diminuir o medo na penetração vaginal.

O presente estudo tem por objetivo, realizar uma revisão integrativa da literatura a respeito das abordagens fisioterapêuticas nas disfunções sexuais femininas. 


\section{Metodologia}

Este estudo, é uma revisão bibliográfica integrativa, com abordagem qualitativa, de modo a proporcionar a síntese de conhecimentos e a incorporação da aplicabilidade dos resultados de estudos, na prática, e de maneira significativa. Embasados em evidências científicas, envolve a definição do problema clínico, bem como a identificação das informações necessárias. A condução da busca de estudos na literatura e sua avaliação crítica e a identificação da aplicabilidade dos dados oriundos das publicações e a determinação de sua utilização para o paciente (Souza et al., 2010).

O estudo foi realizado através de levantamento bibliográfico, em seis fases do processo de produção:

$1^{\circ}$ ) Elaboração da pergunta norteadora: para definir quais serão os descritores utilizados, os estudos incluídos, e a hierarquização das informações coletadas e selecionadas para o estudo.

$2^{\circ}$ ) Busca ou amostragem na literatura: busca ampla e diversificada em base de dados, contemplando a procura em bases eletrônicas, busca manual em periódicos e as referências descritas nos estudos selecionados.

$3^{\circ}$ ) Coleta de dados: com a utilização de um instrumento previamente elaborado capaz de assegurar que a totalidade dos dados relevantes seja extraída, minimizando o risco de erros na transcrição, garantindo precisão na checagem das informações e servindo como registro.

$4^{\circ}$ ) Análise crítica dos estudos incluídos: abordagem organizada para ponderar o rigor e as características de cada estudo.

$5^{\circ}$ ) Discussão dos resultados: a partir da interpretação e síntese dos resultados, comparam-se os dados evidenciados na análise dos artigos ao referencial teórico, identificando possíveis lacunas do conhecimento, delimitando prioridades para estudos futuros, e salientado as conclusões e inferências, bem como explicitando os vieses.

$6^{\circ}$ ) Apresentação da revisão integrativa: após extração dos dados das fontes primárias, mediante utilização de um instrumento elaborado, para simplificar, resumir e organizar os achados. Segue-se a redução, exposição e comparação, bem como a verificação e elaboração da conclusão, a partir dos dados, para apresentação da revisão de forma clara e completa, no artigo final.

A pesquisa foi embasada com referências pesquisadas na plataforma do Google Acadêmico, Scielo, Lilacs, Bireme (Biblioteca Virtual em Saúde-BVS), incluindo artigos e livros com a temática abordada, nos meses de janeiro a setembro de 2021. Os descritores utilizados para a busca foram os seguintes: disfunção sexual feminina, condutas fisioterapêuticas, sexualidade feminina. Critério de Inclusão: artigos publicados em revistas indexadas, nos bancos de dados e site de busca que abordem o tema: terapêutica nas disfunções sexuais femininas, disfunção sexual feminina, condutas fisioterapêuticas, sexualidade feminina; artigos publicados preferencialmente a partir dos últimos onze anos. Critério de exclusão: artigos que não fizeram jus aos critérios de inclusão; artigos que não abordaram especificamente as disfunções sexuais femininas; artigos que não abordaram intervenções fisioterapêuticas nas disfunções sexuais femininas; artigos que foram superiores a onze anos de publicação; artigos que não estabeleceram relevância com o tema proposto. As buscas foram realizadas no período de janeiro a setembro de 2021.

Foram encontrados 43 trabalhos publicados entre livros, revistas e artigos científicos, sendo selecionados 9 artigos como consta na figura 1, por apresentar maior aproximação com os objetivos propostos neste estudo com período de publicação a partir de 2010 a 2021. 
Figura 1- O fluxograma de busca e rastreio das publicações para o presente estudo, exposto abaixo, subsidia este estudo.

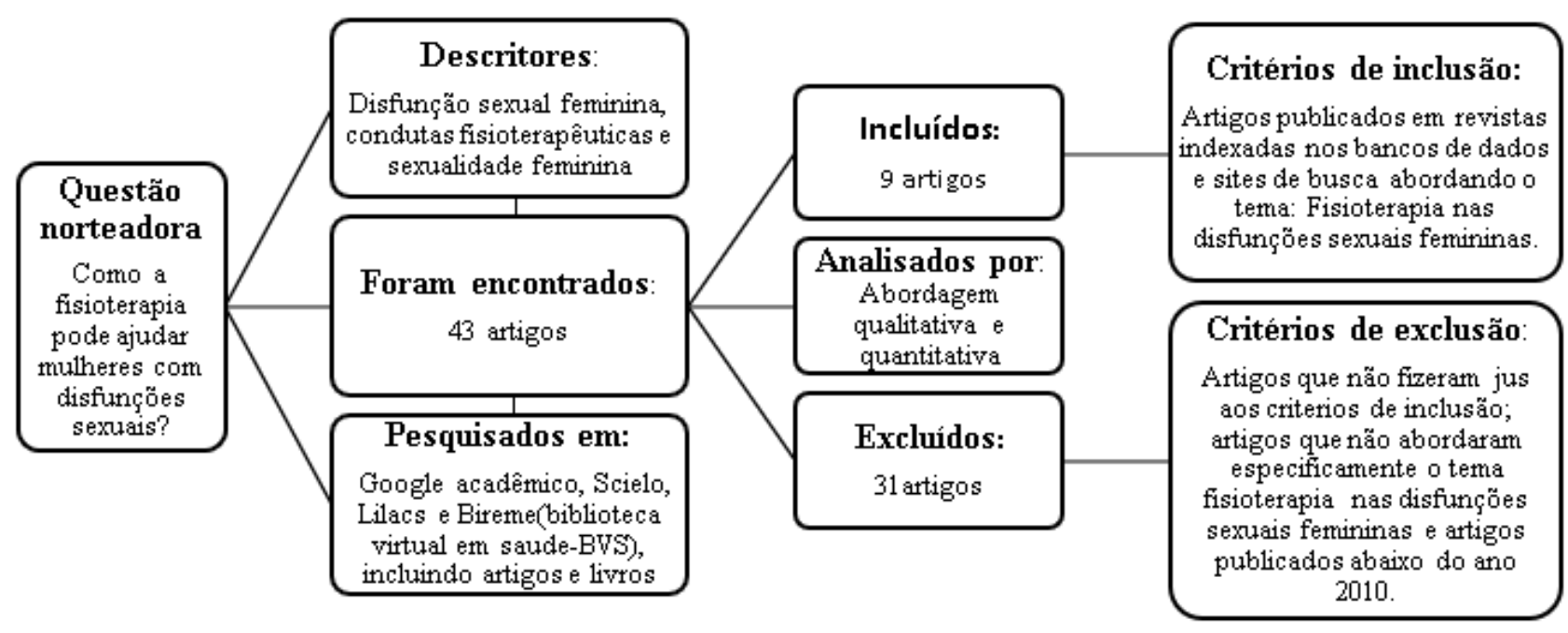

Fonte: Autores (2021).

\section{Resultados e discussão}

As disfunções sexuais femininas são consideradas um problema de saúde pública pela Organização Mundial da Saúde (OMS). Diante dessa abordagem existem diversos estudos que proporcionam um vasto acervo de publicações. Foi elaborada uma tabela (Quadro 1) com 09 artigos selecionados, de acordo com os parâmetros: (1) Nome do artigo; (2) Autor (es); (3) Ano de publicação e (4) Objetivo. 
Quadro 1- Caracterização dos artigos científicos segundo informações como: Nome do artigo, Autor(es), Ano de publicação e Objetivo.

\begin{tabular}{|c|c|c|c|}
\hline Nome do artigo & Autor (es) & Ano & Objetivo \\
\hline $\begin{array}{lrr}\text { Disfunções } & \text { sexuais } & \text { femininas: } \\
\text { questionários } & \text { utilizados } & \text { para } \\
\text { avaliação } \\
\text { inicial. }\end{array}$ & LIMA et al. & 2010 & $\begin{array}{l}\text { Mostrar a importância da fisioterapia ginecológica e atuação } \\
\text { do fisioterapeuta no tratamento das disfunções. }\end{array}$ \\
\hline $\begin{array}{l}\text { Abordagem fisioterapêutica nas disfunções } \\
\text { sexuais femininas }\end{array}$ & $\begin{array}{l}\text { ANTONIOLI R, } \\
\text { SIMÕES }\end{array}$ & 2010 & $\begin{array}{l}\text { Descrever as principais alternativas fisioterapêuticas que } \\
\text { visam minimizar as disfunções sexuais mais encontradas nas } \\
\text { mulheres }\end{array}$ \\
\hline $\begin{array}{l}\text { Recursos Fisioterapêuticos Utilizados No } \\
\text { Tratamento Das Disfunções Sexuais } \\
\text { Femininas }\end{array}$ & DELGADO et al. & 2014 & $\begin{array}{l}\text { Investigar quais recursos fisioterapêuticos são utilizados nos } \\
\text { tratamentos das disfunções sexuais femininas. }\end{array}$ \\
\hline $\begin{array}{l}\text { Tratamento Fisioterapêtico nos } \\
\text { Transtornos Sexuais Dolorosos Femininos }\end{array}$ & LIMA et al. & 2016 & $\begin{array}{l}\text { Analisar na literatura disponível os recursos } \\
\text { fisioterapêuticos utilizados no tratamento dos transtornos } \\
\text { sexuais dolorosos femininos. }\end{array}$ \\
\hline Disfunção sexual em mulheres & $\begin{array}{l}\text { DAS VIRGENS et } \\
\text { al. }\end{array}$ & 2016 & $\begin{array}{l}\text { Discussão sobre as disfunções sexuais femininas e como } \\
\text { esse problema pode influenciar no dia a dia das mulheres. }\end{array}$ \\
\hline $\begin{array}{l}\text { Intervenção da Fisioterapia uroginecológica } \\
\text { no tratamento coadjuvante do vaginismo }\end{array}$ & AMARAL et al. & 2017 & $\begin{array}{l}\text { Verificar o mecanismo de ação da fisioterapia } \\
\text { uroginecológica no tratamento do vaginismo. }\end{array}$ \\
\hline $\begin{array}{l}\text { Atuação do fisioterapeuta nas disfunções } \\
\text { sexuais femininas. }\end{array}$ & $\begin{array}{l}\text { TRINDADE BS \& } \\
\text { LUZES R. }\end{array}$ & 2017 & $\begin{array}{l}\text { Analisar na literatura disponível os recursos } \\
\text { fisioterapêuticos utilizados no tratamento dos transtornos } \\
\text { sexuais dolorosos femininos. }\end{array}$ \\
\hline $\begin{array}{l}\text { Intervenções fisioterapêuticas no tratamento } \\
\text { da dispareunia feminina: um estudo } \\
\text { exploratório. }\end{array}$ & $\begin{array}{l}\text { NETO F.S \& } \\
\text { JERICÓ A.P. }\end{array}$ & 2020 & $\begin{array}{l}\text { Analisar e descrever com uma boa qualidade metodológica } \\
\text { a abordagem da fisioterapia no tratamento da dispareunia } \\
\text { feminina. }\end{array}$ \\
\hline $\begin{array}{l}\text { Fisioterapia na anorgasmia feminina: Uma } \\
\text { revisão integrativa. }\end{array}$ & $\begin{array}{l}\text { PAVANELO D.D } \\
\text { et al. }\end{array}$ & 2020 & $\begin{array}{l}\text { Descrição, comparação e análise crítica de sete questionários } \\
\text { validados utilizados na avaliação das Disfunções Sexuais } \\
\text { Femininas (DSF). }\end{array}$ \\
\hline
\end{tabular}

Fonte: Autores (2021).

De acordo com Lima et al., (2010), entende-se por disfunção sexual feminina o comprometimento através de bloqueio ou inibição em qualquer uma das três fases do ciclo de resposta sexual (1) desejo, (2) excitação e (3) orgásmica. As causas determinantes desse distúrbio poderão estar ligadas à própria estrutura orgânica do indivíduo (neurológicas, hormonais ou outras) ou a influências psicoculturais, que mutilem ou distorçam a funcionalidade sexual.

Já para o autor Das Virgens et al., (2016), as disfunções em mulheres podem ocorrer em qualquer faixa etária, porém não são bem diagnosticadas, pois as pacientes não se queixam devido à inibição, o médico não investiga por constrangimento ou por não ter um entendimento sobre a resposta sexual humana. Ainda conforme o mesmo autor, as patologias sexuais que são de conhecimento do ginecologista são muito restritas, e muitas vezes, esbarra na falta de conhecimento da anatomia genital e da resposta sexual humana.

Lima et al., (2010) discorre que nos distúrbios de disfunção sexual incluem-se as seguintes categorias: distúrbios do desejo sexual (desejo sexual hipoativo e aversão sexual), distúrbios da excitação sexual, anorgasmia e distúrbios de dor durante 
o ato sexual (dispareunia, sendo a dor durante o ato sexual), vaginismo em que há um reflexo involuntário ou precipitado por tentativas reais, ou imaginárias de penetração vaginal e a dor sexual não coital quando a dor é somente por estímulos do desejo sexual, onde não acontece a penetração). Antonioli e R Simões (2010), ainda acrescentam a dor pélvica crônica, caracterizada por uma dor constante com mais de três meses de evolução, um tipo de dor intermitente que se apresenta por mais de seis meses, incluindo a dispareunia profunda e a dismenorreia; e a anorgasmia secundária que é a falta de orgasmo em consequência de algum problema biopsicossocial.

A fisioterapia vem ganhando um papel muito importante na equipe multidisciplinar quando se refere ao tratamento relacionado a saúde da mulher. E a fisioterapia ginecológica é uma área ainda pouco conhecida entre os pacientes e profissionais da área de saúde, mas vem crescendo cada dia mais (Trindade et al., 2017).

Os fisioterapeutas são responsáveis pela avaliação, diagnóstico, educação, tratamento, e por fornecer informações anatômicas da região genital das pacientes; o tratamento fisioterapêutico não precisa do acompanhamento integral do fisioterapeuta, desde que haja o entendimento da paciente sobre sua condição e a colaboração para o tratamento. (Pavanelo et al., 2021). Barbosa (2016), acrescenta ainda que o tratamento fisioterapêutico inclui anamnese da paciente, com inspeção visual e palpação do assoalho pélvico, identificação das condições da musculatura, pontos de dor, presença de incontinências urinária, fecal e flatos, distopias, testes de sensibilidade (táctil, térmica e dolorosa) e reflexos na região pélvica e do assoalho pélvico.

Os métodos fisioterapêuticos utilizados baseiam-se na contração voluntária dos músculos perineais para reeducar o assoalho pélvico e aumentar seu tônus muscular. A aplicação dos protocolos de tratamento difere consideravelmente, mas a magnitude das resistências aplicadas, as durações do tempo de contração e de repouso, dependem também, de uma posição correta da pelve e de uma respiração normal (Neto \& Jericó, 2020).

Para o autor Barbosa, (2016) a fisioterapia vem ainda se destacando no tratamento dos transtornos sexuais dolorosos das mulheres, através da utilização de múltiplas técnicas de reabilitação neuromuscular, entre elas a eletroestimulação, o biofeedback, a cinesioterapia e o trabalho manual.

Já Pavanelo et al., (2020) explica que os melhores resultados são demonstrados com o uso de alguns recursos como parte do plano de tratamento da fisioterapia, quando há uma disfunção, tais como: Cinesioterapia para o treinamento da musculatura do assoalho pélvico (TMAP), o Método Pilates associado à contração da musculatura do assoalho pélvico (MAP) e à Eletroestimulação. Segundo esse autor, esses recursos e técnicas se mostram promissoras no tratamento das disfunções sexuais femininas, a prescrição dos exercícios pelo fisioterapeuta precisa ainda, ser baseada na anamnese e na avaliação física minuciosa, devendo ser considerado fatores biopsicossociais.

Segundo Pavanelo et al., (2020) em análise, no que se refere aos recursos e técnicas fisioterapêuticas mais utilizados no tratamento das disfunções, temos a cinesioterapia, trabalho manual, biofeedback e eletroestimulação.

\section{Cinesioterapia}

Antonioli e R Simões (2010), explica que a cinesioterapia do assoalho pélvico é em maioria executada através dos exercícios de Kegel, que consiste em exercícios perineais supervisionados, podendo ser feita em associação à respiração abdominal-diafragmática seguindo instruções verbais do terapeuta, cada contração pode ser realizada em 5 segundos com séries de 8 repetições em diferentes posições, ou mais brevemente de 1 a 2 segundos. Seu objetivo é estimular a musculatura perineal para o tratamento de hipotonia, melhorando a sustentação pélvica e resistência uretral, além de ter considerável importância, pois sugere a reabilitação da musculatura pélvica através de contrações isoladas com posicionamento correto da pelve e respiração apropriada. Com diferentes posturas torna-se fácil o recrutamento de músculos específicos, aumentando o controle e a consciência da mulher. Esses exercícios têm elevada importância em DSF como o vaginismo, dor pélvica, dispareunia e a anorgasmia por promover controle da musculatura pélvica, força e melhor propriocepção. (Delgado et al., 2014) 


\section{Trabalho manual}

O Trabalho manual é uma grande aliada no tratamento das DSF, dentre suas principais técnicas estão: A massagem perineal que promove melhor fluxo sanguíneo, relaxamento, alívio de dores e normalização do tônus. E o toquebigital onde o fisioterapeuta insere dois dedos (o médio e o indicador) na vagina da paciente, até a região onde deve contrair. Após a localização da musculatura que se pretende recrutar, o fisioterapeuta estende os dedos para estimular a força de contração positiva. Podendo ser graduado em: sem contração, quando o músculo se encontra sem força, pouca contração, a força da musculatura não vence a resistência provocada pelo estímulo e tem contração quando a musculatura reage ao estímulo provocado. Variando 0 a 3 . Através desta terapia é possível melhorar a percepção corporal e relaxamento muscular durante o ato sexual. Essas duas técnicas auxiliam significativamente em acometimentos como a dor pélvica, vaginismo e a dispareunia. (Delgado et al., 2014; Antonioli \& R Simões, 2010).

\section{Biofeedback}

Segundo Amaral (2017), o Biofeedback é considerado um aparelho digital que mede, avalia e trata as disfunções neuromusculares, responsável em avaliar o assoalho pélvico onde acompanha o tônus em repouso, força e sustentação. Desse modo, é possível orientar mulheres sobre a respectiva melhora tanto das contrações voluntárias da musculatura quanto do assoalho pélvico, beneficiando assim o relaxamento. O objetivo do tratamento por biofeedback é de ajudar as pacientes a desenvolver maior percepção e controle voluntário dos músculos do assoalho pélvico. Sua contribuição consiste também em garantir a aquisição rápida, precisa, segura da participação da paciente em sua reeducação sendo uma técnica útil em disfunções como o vaginismo, anorgasmia e vulvodínia. (Antonioli \& R Simões, 2010).

\section{Eletroestimulação}

A eletroestimulação realizada através do TENS (eletroestimulação transcutânea) e o FES (Estimulação elétrica funcional) propiciam potentes estímulos elétricos na área pudenda. Com suas respectivas funções esses recursos têm bastante eficácia no que diz respeito ao efeito analgésico pela liberação de endorfina, conscientização dos músculos do assoalho pélvico, reforço muscular e liberação muscular, auxiliando em patologias como vaginismo, dor pélvica, vulvodínia e dispareunia, no entanto, a corrente elétrica precisa ser ajustada a um ponto que possa ser sentida, mas que não seja desconfortável para o paciente. (Lima 2016; Antonioli \& R Simões, 2010).

\section{Considerações Finais}

Os artigos pesquisados apresentaram resultados promissores diante da atuação do fisioterapeuta. Todos eles, comprovam que a fisioterapia junto a equipe multidisciplinar, possui um papel fundamental no tratamento e na recuperação das disfunções sexuais. Embora ainda pouco explorada o estudo se mostrou promissor, destacando a cinesioterapia, trabalho manual, biofeedback e a eletroestimulação como precursor de efeitos positivos no tratamento de anorgasmia, dispareunia, vaginismo, vulvodínia, dor pélvica crônica, aumento da excitação e desejo sexual. Apesar da escassez de estudos que comparem duas ou mais terapêuticas para melhorar a prática baseada em evidências, nesse estudo mostrou-se a eficácia dos recursos fisioterapêuticos nas disfunções sexuais femininas. As técnicas e propedêuticas utilizadas, se dão em sua maioria para fortalecimento, conscientização, analgesia, controle e reeducação tanto da musculatura perineal, quanto a do assoalho pélvico, além disso, apresentou a capacidade das condutas fisioterapêuticas de promover maior qualidade de vida e satisfação sexual. Durante o estudo foi observado uma abordagem desatualizada por parte de alguns profissionais que atuam na área, pois a atual é insuficiente na comunicação e informação para as pacientes, o que acaba gerando insegurança, reprimindo e ocultando sintomas 
e dificultando um diagnóstico preciso e o tratamento adequado. São necessários estudos de campo e aprofundamento na área atualizados, para possibilitar mais clareza aos profissionais e segurança para orientar a paciente de forma correta, aperfeiçoando assim o diagnóstico e o tratamento.

\section{Referências}

Amaral, P. P. (2017). Intervenção da fisioterapia uroginecológica no tratamento coadjuvante do vaginismo. Revista Visão Universitária, 2(1).

Antonioli, R. de S., \& Simões, D. (2010). Abordagem fisioterapêutica nas disfunções sexuais femininas. Revista Neurociências, 18(2), $267-274$.

Costa, L. H. R., \& Coelho, E. C. D. A. (2011). Enfermagem e sexualidade: revisão integrativa de artigos publicados na Revista Latino-Americana de Enfermagem e na Revista Brasileira de Enfermagem. Revista Latino-Americana de Enfermagem, 19, 631-639.

Das Virgens, G. D., Bernardes, F. C., Santos, G. D., de Freitas, C. C., \& Gomes, E. M. R. (2016). Disfunção Sexual em Mulheres. Revista Eletrônica Acervo Saúde, (3), 119-125.

De Castro, K. M. S. (2020) Fisioterapia na disfunção sexual feminina: uma revisão.

Delgado, A. M., Ferreira, I. S. V., \& de Sousa, M. A. (2014). Recursos fisioterapêuticos utilizados no tratamento das disfunções sexuais femininas. CATUSSABAISSN 2237-3608, 4(1), 47-56.

Dias, J. C., dos Santos, W. S., de Sousa Pereira, J., \& de Vasconcelos, R. F. R. (2015). Anorgasmia Feminina. Revista Interfaces: Saúde, Humanas e Tecnologia, $2(6)$.

Felipe, A. P. (2020) Atuação da fisioterapia na dor pélvica crônica feminina: uma revisão.

Fleury-Teixeira, E., \& Meneghel, S. N. (Eds.). (2015). Dicionário Feminino da Infâmia: acolhimento e diagnóstico de mulheres em situação de violência. SciELO-Editora FIOCRUZ.

Latorre, G. F. S., Manfredini, C. C., Demeterco, P. S., Barreto, V. M. N. F., \& Feio Carneiro Nunes, E. (2015). A fisioterapia pélvica no tratamento da vulvodínia: revisão sistemática. Femina, 257-264

Lima, S. M. R. R., dos Santos Silva, H. F., Postigo, S., \& Aoki, T. (2010). Disfunções sexuais femininas: questionários utilizados para avaliação inicial. Arquivos Médicos dos Hospitais e da Faculdade de Ciências Médicas da Santa Casa de São Paulo, 1-6.

Lima, R. G. R., dos Santos Silva, S. L., da Boaviagem Freire, A., \& Barbosa, L. M. A. (2016). Tratamento fisioterapêutico nos transtornos sexuais dolorosos femininos: revisão narrativa. Revista Eletrônica da Estácio Recife, 2(1).

Milanese, C. V. S. (2020). As influências do feminismo na sexualidade das mulheres brasileiras. Estudos em Sexualidade Volume 2 , 90.

Moreira, R. L. B. D. (2013). Vaginismo.

Neto, F. S., \& Jericó, A. L. P. (2020). Intervenções fisioterapêuticas no tratamento da dispareunia feminina: um estudo exploratório. Research, Society and Development, 9(9), e209996570-e209996570.

Pavanelo, D. D., \& Dreher, D. Z. (2021, July). Fisioterapia na anorgasmia feminina: uma revisão integrativa. In Congresso Internacional em Saúde (No. 8).

Ribeiro, B., Magalhães, A. T., \& Mota, I. (2013). Disfunção sexual feminina em idade reprodutiva: prevalência e factores associados.

Soares, E. R. (2013). Disfunção sexual feminina: Tratamento fisioterapêutico na dispareunia.

Souza, L. C., Pereira, E. C. A., Vasconcelos, E. F. S., \& Pereira, W. M. P. (2020). Fisioterapia na disfunção sexual da mulher: revisão sistemática. Revista Ciência e Saúde On-line, 5(2).

Souza, M. T. D., Silva, M. D. D., \& Carvalho, R. D. (2010). Revisão integrativa: o que é e como fazer. Einstein (São Paulo), 8, $102-106$.

Trindade, S., \& Luzes, R. (2017). Atuação do fisioterapeuta nas disfunções sexuais femininas. Alumni-Revista Discente da UNIABEU-ISSN 2318-3985, 5(9), 10-16. 